\title{
Animal-Free Human Whole Blood Sepsis Model to Study Changes in Innate Immunity
}

\author{
David Alexander Christian Messerer ${ }^{1,2 *}$, Laura Vidoni ${ }^{1}$, Maike Erber ${ }^{1}$, \\ Alexander Elias Paul Stratmann ${ }^{1}$, Jonas Martin Bauer ${ }^{1}$, Christian Karl Braun ${ }^{1}$, \\ Stefan Hug ${ }^{1}$, Anna Adler ${ }^{3}$, Kristina Nilsson Ekdahl ${ }^{3,4}$, Bo Nilsson ${ }^{3}$, \\ Eberhard Barth ${ }^{2}$, Peter Radermacher ${ }^{5}$ and Markus Huber-Lang ${ }^{1}$
}

${ }^{1}$ Institute of Clinical and Experimental Trauma Immunology, University Hospital of UIm, UIm, Germany, ${ }^{2}$ Department of Anesthesiology and Intensive Care Medicine, University Hospital of Ulm, Ulm, Germany, ${ }^{3}$ Rudbeck Laboratory, Department of Immunology, Genetics and Pathology, Uppsala, Sweden, ${ }^{4}$ Centre of Biomaterials Chemistry, Linnaeus University, Kalmar, Sweden, ${ }^{5}$ Institute for Anesthesiologic Pathophysiology and Process Engineering, Ulm University, Ulm, Germany
OPEN ACCESS

Edited by:

Fabrice Cognasse,

Groupe Sur L'immunité Des Muqueuses Et Agents Pathogènes (GIMAP), France

Reviewed by:

Charles E. McCall, Wake Forest Baptist Medical Center,

United States

Rafael Agustín Burgos,

Austral University of Chile, Chile

${ }^{*}$ Correspondence: David Alexander Christian Messerer david.messerer@uni-ulm.de

Specialty section: This article was submitted to Inflammation,

a section of the journal

Frontiers in Immunology

Received: 12 June 2020 Accepted: 21 September 2020 Published: 14 October 2020

Citation:

Messerer DAC, Vidoni L, Erber M, Stratmann AEP, Bauer JM, Braun CK, Hug S, Adler A, Nilsson Ekdahl K, Nilsson $B$, Barth $E$, Radermacher $P$ and Huber-Lang M (2020) Animal-Free Human Whole Blood Sepsis Model to Study Changes in Innate Immunity.

Front. Immunol. 11:571992. doi: 10.3389/fimmu.2020.571992
Studying innate immunity in humans is crucial for understanding its role in the pathophysiology of systemic inflammation, particularly in the complex setting of sepsis. Therefore, we standardized a step-by-step process from the venipuncture to the transfer in a human model system, while closely monitoring the inflammatory response for up to three hours. We designed an animal-free, human whole blood sepsis model using a commercially available, simple to use, tubing system. First, we analyzed routine clinical parameters, including cell count and blood gas analysis. Second, we demonstrated that extracellular activation markers (e.g., CD11b and CD62I) as well as intracellular metabolic (intracellular $\mathrm{pH}$ ) and functional (generation of radical oxygen species) features remained stable after incubation in the whole blood model. Third, we mimicked systemic inflammation during early sepsis by exposure of whole blood to pathogen-associated molecular patterns. Stimulation with lipopolysaccharide revealed the capability of the model system to evoke a sepsis-like inflammatory phenotype of innate immunity. In summary, the presented model serves as a convenient, economic, and reliable platform to study innate immunity in human whole blood, which may yield clinically important insights.

Keywords: inflammation, neutrophil granulocytes, lipopolysaccharide (LPS), blood physiology, sepsis, ex vivo whole blood model, principles of the 3Rs

\section{INTRODUCTION}

Innate immunity plays a vital role as the vanguard against numerous harmful carriers of damage- and pathogen associated molecular patterns (DAMPs and PAMPs, respectively). An appropriate inflammatory response efficiently clears pathogens and initiates subsequent healing processes. However, an excessive and dysregulated inflammation is a matter of both increasing interest and concern, particularly during trauma (1) and sepsis (2). A recent study estimated the annual global incidence of sepsis at approximately 50 million (3). Moreover, the latest consensus 
definition of sepsis underscored the importance of appropriate regulated (innate) immunity in the successful clearance of pathogens (2).

To study the complex innate immune response to PAMP exposure, various small or large, simple or complex animal models are used (4-6). However, ethical considerations, including the aspect of replacement within the 3R-principles (7), possible limitations in translation into real-world patients (4, 5), and the expense of personal and material resources, may limit the validity and attractiveness of animal-based models. In addition, whole blood samples or tissue specimens of patients suffering from sepsis can only be obtained at the cost of additional burden and risk to the patients. Moreover, the samples originate from different etiological, microbiological, and genetic backgrounds, and thus cannot be standardized precisely. For example, the exact exposure time and the amount of pathogens within the blood during sepsis cannot be determined with certainty. Therefore, as another alternative, injection of immune-stimulants, including lipopolysaccharide (LPS), has been performed and investigated in healthy human volunteers $(8-10)$. On the one hand, this approach provides the intriguing opportunity to study the immunological response directly in human organisms. On the other hand, obvious ethical considerations limit the possibilities of this method, for example, constraining the severity of the induced inflammation or being restricted to young and healthy individuals (9). However, because excessive inflammation during sepsis remains a major clinical and scientific challenge, there is an unabated research need to elucidate the underlying immunological pathomechanisms, for example, by utilizing human whole blood in a standardized and reliable manner.

Exposure of blood to DAMPs, PAMPs, and/or defined pathogens outside the human body surmounts several limitations indicated above. Notwithstanding, there are some cellular and humoral components in the blood that become rapidly activated when losing their contact with intact endothelium, which under homeostatic conditions acts as an anti-inflammatory, antiadhesive, and anti-coagulatory regulator. In particular, platelets and serine cascades are controlled by an intact endothelium (11). To address the issue of "hemoincompatibility" of artificial materials, whole blood is frequently completely anticoagulated, for example, either by heparin-based anticoagulants or cationchelators, including citrate, both of which can alter the ability of humoral and cellular immunity to adequately respond to stimulation.

To prevent stimulation after venipuncture ex vivo, a variety of models have been previously proposed of whole blood being supplemented with an anticoagulant and/or being encased in a specially coated system. Human whole blood models of coated circuits or whole blood cultures in combination with defined anticoagulation, for example, heparin or hirudin, have been widely used and characterized in recent years (12-18). In general, these models address a specific focus and produced the respective specific answers. For example, they were used to elucidate the interaction of whole blood stimulated with various bacteria, revealing the significance of the complement factors $3 \mathrm{a}$ and $5 \mathrm{a}(\mathrm{C} 3 \mathrm{a}, \mathrm{C} 5 \mathrm{a})$ as well as $\mathrm{CD} 14$ for the crosstalk of pathogens and leukocytes (12-15).

However, to our knowledge, the description of hitherto existing models focused on the characterization of humoral immunity and other protease systems such as the coagulation cascade, without elucidating the impact of exposition to an ex vivo circuit to general (patho-)physiological and metabolic responses in association with cellular innate immunity. For example, global blood parameters (e.g. $\mathrm{pH}$ and glucose concentration) have frequently not been reported, making it difficult to interpret the presented data and to transfer them into the physiological in vivo context of the human body $(12-14,16$, $18-21)$. There is also a concomitant research gap with regards to a comprehensive study of blood physiology ex vivo while synchronically assessing important immunological functions and activation markers.

Here, we 1) standardized the handling of an easy-to-use whole blood model, 2) determined a myriad of global blood parameters with a comprehensive focus on extra- and intracellular parameters of innate immunity, and 3) mimicked septic conditions and subsequent systemic inflammation in blood in an animal-free research setup by stimulation with LPS.

\section{METHODS}

\section{Blood Sampling}

Healthy human volunteers of both genders aged 21-30 years served as blood donors. All experiments were conducted in accordance with the declaration of Helsinki (22). Following ethical approval (number 459/18, Local Independent Ethics Committee of the University of Ulm) and written informed consent, blood was drawn by peripheral venipuncture in accordance with the guidelines of the World Health Organization (23). In detail, blood stasis was limited to a maximum of $30 \mathrm{~s}$ before puncture and the first $2-3 \mathrm{ml}$ were immediately discarded. The blood was collected in neutral monovettes (Sarstedt, Nürnbrecht, Germany), which had been supplemented with heparin (B. Braun Melsungen AG, Melsungen, Germany) and either LPS (Sigma Aldrich, Steinheim, Germany) or phosphate-buffered saline $\left(\mathrm{PBS}^{++}\right.$, as control), respectively. In total, $9 \mathrm{ml}$ blood was collected per tube, with a final concentration of $0.5 \mathrm{IU} / \mathrm{ml}$ of heparin and when present, $100 \mathrm{ng} / \mathrm{ml}$ of LPS.

\section{Whole Blood Model}

Immediately after sampling, the blood was transferred carefully using $10 \mathrm{ml}$ tips (Eppendorf, Hamburg, Germany) into the following described system. The whole blood model consisted of a heparin coated tubing system (Cortiva, \#M999413C, Medtronic, Meerbusch, Germany), being cut into approximately $33 \mathrm{~cm}$ long pieces. Both ends of the tube were added to a circuit using a similarly coated connector (Cortiva, \#CB4629, Medtronic), leaving an air bubble of approximately $1.5 \mathrm{ml}$ inside the system. The loops were attached to a spinning wheel (Snijders Labs, Tilburg, Netherlands) rotating at $5 \mathrm{rpm}$, causing the air bubble 
to generate a continuous circulation of the blood. As described previously, the combination of heparin-coating and continuous circulation mediated by a small air bubble allows incubating whole blood with a low-dose of heparin (24). Another option would be the application of higher doses of heparin, however, this interferes with important immunological systems such as the complement system (24) or the leukocytes (25). The authors actively decided against to use a mechanic pump, as this causes mechanical stress, that among other consequences results in hemolysis and platelet activation (26).

The system was incubated for 0 (depicted as " 0 " indicating no contact with the tubing system), 10, 60, or $180 \mathrm{~min}$ as indicated in an incubator at $37^{\circ} \mathrm{C}$ without additional $\mathrm{CO}_{2}$. Following the incubation period, the tubing loops were cut open. Initially, $95 \mu$ l blood were directly drawn into dry-heparin anticoagulated glass capillary tubes (Radiometer $\mathrm{GmbH}$, Krefeld, Germany). Subsequently, $1 \mathrm{ml}$ of the blood was transferred into a heparin anticoagulated tube (Sarstedt) for the analysis of phagocytotic activity and radical generation. The remaining blood was transferred into citrate anticoagulated monovettes (Sarstedt) for the analysis of all other parameters. Sodium, potassium, ionized calcium, lactate, glucose, and blood $\mathrm{pH}$ were determined using a standard blood gas analyzer (ABL 800 Flex, Radiometer $\mathrm{GmbH}$ ). To calculate the difference in the amount of lactate generated or the consumption of glucose, the respective value after $60 \mathrm{~min}$ with or without incubation with LPS was subtracted from the corresponding baseline $\left(0^{-}\right)$. Differential blood count and global coagulation parameters (activated partial thromboplastin time, aPTT, and international normal ratio (INR)) were determined using a standard hematology (Sysmex CN 2000, Sysmex, Kobe, Japan) and coagulation (BCS XP, Siemens, Marburg, Germany) analyzer, respectively, each according to the manufacturer's standard protocol.

\section{Flow Cytometry}

For antibody staining, $10 \mu \mathrm{l}$ citrate anticoagulated blood were added to $40 \mu \mathrm{PBS}^{++}$adjusted to $\mathrm{pH} 7.3$ and stimulated with 100 ng/ml C5a (Complement Technology, Tyler, Texas, USA) or PBS as control for $15 \mathrm{~min}$ in a water bath at $37^{\circ} \mathrm{C}$. Subsequently, the cells were stained as indicated with anti-CD11b (APC, dilution 1:82 \#101212, BioLegend, San Diego, California, USA), antiCD14 (APC-Cy7, dilution 1:200 \#301820, BioLegend), anti-CD621 (PE, dilution 1:33 \#304806, BioLegend), anti-CD88 (APC, dilution 1:250 \#344310, BioLegend), or corresponding isotype controls (all from BioLegend), respectively, for $15 \mathrm{~min}$ at room temperature. All markers were assessed by monoclonal mouse anti-human antibodies. Cellular viability was analyzed by identifying necrotic cells with the Zombie Violet Fixable Viability Kit (dilution 1:4000, \#423114, BioLegend) and apoptotic cells using Apotracker Green (final concentration $200 \mathrm{nM}$, \#427402, BioLegend).

The generation of radical oxygen species (ROS) was determined by staining $40 \mu \mathrm{l}$ heparin anticoagulated blood with $0.29 \mathrm{mM}(100 \mu \mathrm{g} / \mathrm{ml})$ dihydrorhodamine 123 (DHR, Santa Cruz Biotechnology, Dallas, Texas, USA). Phagocytosis was analyzed using fluorescent microspheres (Fluoresbrite ${ }^{T M}$ Carboxylate YG
0.75 Microspheres, Polysciences, Inc., Warrington, Pennsylvania, USA). The microspheres were dissolved 1:20 in $\mathrm{PBS}^{++}$followed by a washing procedure ( $3 \times$ at $1000 \mathrm{~g}$ for $5 \mathrm{~min}$ ). Of this microsphere solution, $10 \mu \mathrm{l}$ was added onto $100 \mu \mathrm{l}$ heparin anticoagulated whole blood. Whole blood stained with either DHR or incubated with microspheres was incubated for 20 and $30 \mathrm{~min}$, respectively, at $37^{\circ} \mathrm{C}$ in the dark. In all experiments, after stimulation and staining of whole blood, the erythrocytes were lysed and the leukocytes fixed by filling up the sample volume to $1 \mathrm{ml}$ with $1 \times$ BD FACS lysing solution ${ }^{\text {TM }}$ (BD Biosciences, San Jose, California, USA) for $30 \mathrm{~min}$ at room temperature in the dark. Following centrifugation of the samples for $5 \mathrm{~min}$ at $340 \mathrm{~g}$, the specimens were resuspended in $100 \mu \mathrm{PBS}+0.1 \%$ bovine serum albumin and stored at $4^{\circ} \mathrm{C}$ until further analysis (normally within $1 \mathrm{~h}$ ).

For the analysis of living granulocytes and plasma, citrate anticoagulated whole blood was centrifuged for $10 \mathrm{~min}$ at $400 \mathrm{~g}$ at room temperature. The supernatant plasma was carefully removed from the hematocrit and stored at $-80^{\circ} \mathrm{C}$ until further use for enzyme-linked immunosorbent assay (ELISA) analysis. The remaining blood cells were subjected to dextran sedimentation followed by hypotonic lysis of the erythrocytes. Forward scatter (FSC) area was used as a surrogate for cellular shape (27). The intracellular $\mathrm{pH}$ was determined using the fluorescent dye SNARF (Thermo Fisher Scientific, Waltheim, Massachusetts, USA) with nigericin-based calibration curves as described previously (28).

For all flow cytometry experiments, neutrophils and monocytes were gated based on their forward and sideward scatter (SSC) area properties. Doublets were removed by plotting forward scatter area versus height. Spillover between fluorescence channels was corrected by a compensation matrix. For all antigens, appropriate isotype controls (see figures) and single-staining controls were performed (data not shown). For all experiments, a minimum of 300 monocytes and 1000 neutrophils were recorded using a FACS Canto II (BD Biosciences).

\section{Determination of Platelet-Neutrophil- Complexes}

Platelet-neutrophil-complexes (PNC) were analyzed by light microscopy (29). In brief, $100 \mu$ citrate anticoagulated blood was diluted with $100 \mu \mathrm{PBS}^{++}$. Blood smears were stained with the "Hemacolor ${ }^{\circledR}$ Rapid staining of blood smear - staining set for microscopy" (Merck KGaA, Darmstadt, Germany). A minimum of 50 neutrophils per specimen were analyzed by two independent and blinded individuals. Each neutrophil with at least one thrombocyte in direct proximity was counted as a PNC.

\section{ELISA}

Determination of plasma levels of C3a, matrix metallopeptidase 9 (MMP9), interleukin 6 (Il6), and interleukin 8 (Il8) was conducted by standard ELISA as indicated by the manufacturer (MMP9: R\&D Systems, Minneapolis, Minnesota, USA; Il6: BD Biosciences; Il8: R\&D Systems; C3a: Quidel Corporation, San Diego, California, USA). 


\section{Data Analysis and Statistics}

All data is presented as median with error bars indicating the interquartile range (IQR). When informative (e.g. to assess donor heterogeneity in cellular immunity), additional scatter plots are presented as overlay. The sampling strategy consisted of an initial experiment series with $\mathrm{n}=3$ for all time points $\left(0^{-}\right.$, $10 \mathrm{~min}, 60 \mathrm{~min}, 180 \mathrm{~min}, 60 \mathrm{~min}$ with LPS, $180 \mathrm{~min}$ with LPS) focusing on blood gas analysis, whole blood count, and humoral inflammation. Because blood glucose, $\mathrm{pH}$, and lactate level became unphysiological beyond $1 \mathrm{~h}$ and previous literature indicated that the neutrophil phenotype as measured by CD11b/CD62l expression reached its final alteration after exposure to LPS for $1 \mathrm{~h}$ (30), cellular immunity was analyzed for this time point from at least five independent specimen. Data analysis was performed with licensed versions of Microsoft Excel 2019 (Microsoft, Redmond, Washington, USA) and GraphPad Prism 8 (GraphPad Software Inc, San Diego, California, USA). In statistical testing, data was considering as paired and nonnormally distributed: For comparison of two groups, a Wilcoxon matched-pairs signed rank test was conducted (Figures 4, 5, comparison of control vs. C5a within the respective condition). Multiple-group comparison was performed by the Friedman test for paired data (Figures 4-6A, B, comparison of $0^{-}$vs. Ctrl vs. LPS) or Kruskal-Wallis test for unpaired data (Figures 6C, D, comparison of $0^{-}$vs. Ctrl vs. LPS), respectively, followed by Dunns' multiple comparison test. A $p$ value $<0.05$ was considered to be significant and marked by ${ }^{*}$ or ${ }^{\star *}$, indicating $<0.05$ or 0.01 , respectively.

\section{RESULTS}

\section{Stability of Global Physiological Parameters in the Ex Vivo Whole Blood Model During the First Hour}

In the present whole blood model, the leukocyte and erythrocyte cell counts remained stable for up to $3 \mathrm{~h}$ after circulation in comparison to baseline values (directly after venipuncture, Figures 1A, B). In accordance, the amount of necrotic and/or apoptotic cells for neutrophils, monocytes, and lymphocytes was determined to be $<2 \%(\mathrm{n}=3$, data not shown). Furthermore, the activity of hemostasis and the coagulation system was monitored: platelet count (Figure 1C) and the formation of PNCs (Figure 1D) indicated no initiation of cellular coagulation. As expected, after the addition of $0.5 \mathrm{IU}$ heparin/ $\mathrm{ml}$ and incubation for $60 \mathrm{~min}$, humoral coagulation was inhibited as indication by an INR of $2.1(1.8 ; 2.3, \mathrm{n}=4)$ and an aPTT of $>180 \mathrm{~s}$ in all four analyzed samples (data not shown). No visible clot formation was detected in any of the analyzed specimens.

Electrolyte concentrations including sodium, potassium, and ionized calcium were stable throughout the 3 -h period of interest (Figures 2A-C). Because the potassium levels did not increase and the red blood cell count and hemoglobin did not decrease, no considerable hemolysis had occurred in the system.

Regarding metabolic changes, there was an expected glucose consumption over time. The glucose concentration (Figure 2D) decreased within the first hour of incubation with a median of -
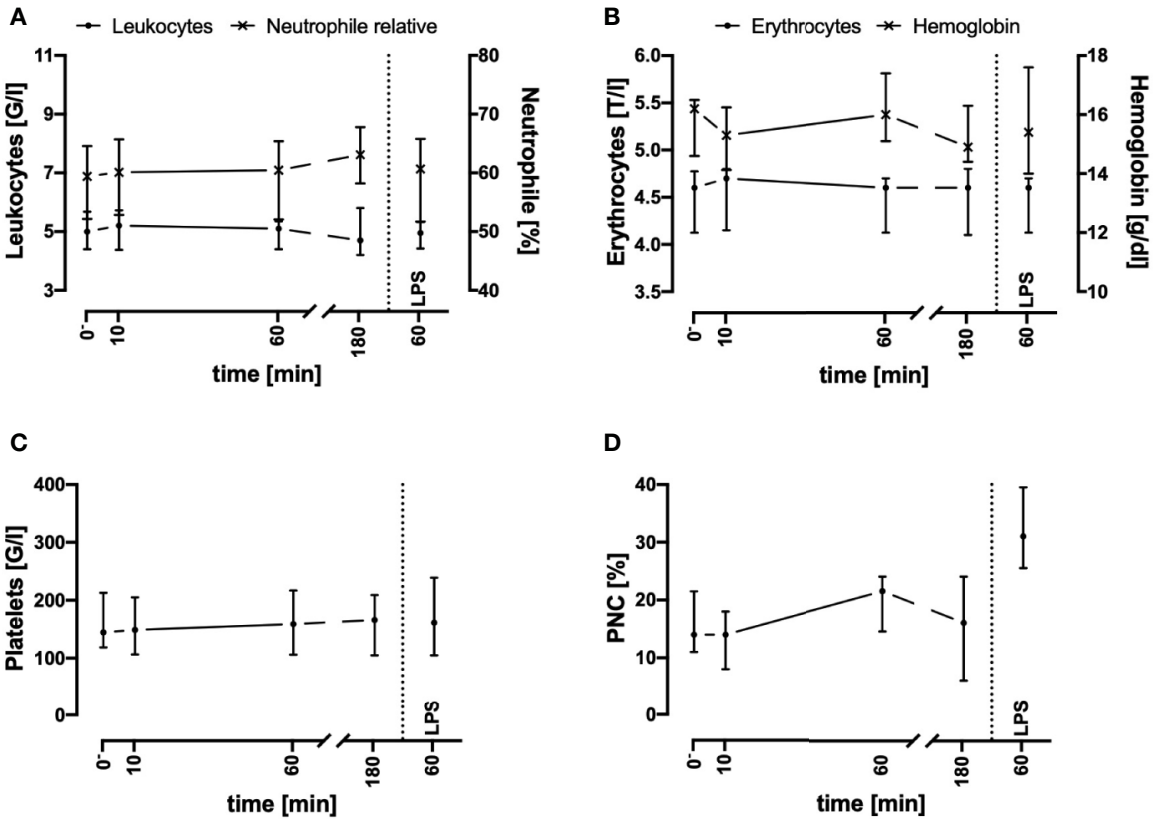

FIGURE 1 | Cell counts of leukocytes (A), erythrocytes (B), platelets (C), and platelet-neutrophil complexes (PNC) (D) before and after contact of blood with the tubing system. Cell counts and PNC formation remained stable during the first $3 \mathrm{~h}$ after exposure of whole blood in the ex vivo model. LPS (100 ng/ml) was used as a potent PAMP stimulus. $n=3$. Results are presented as median with error bars indicating the interquartile range. 


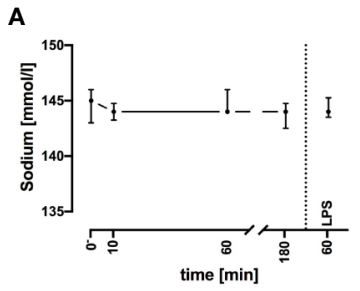

D

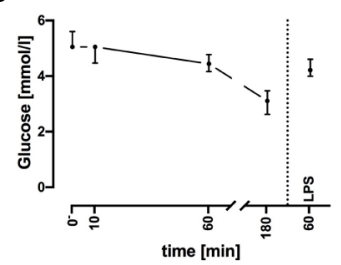

G

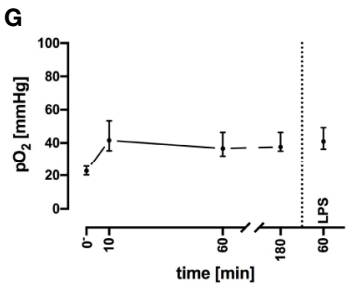

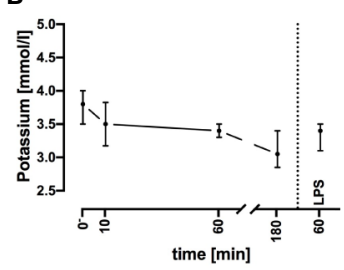

E

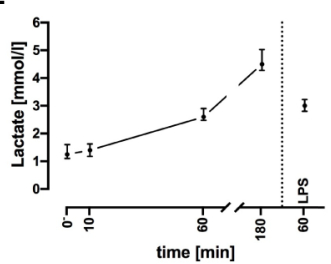

H

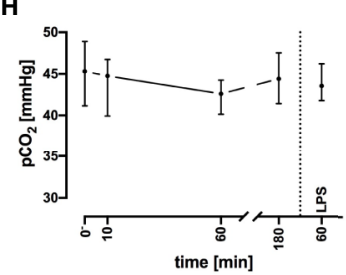

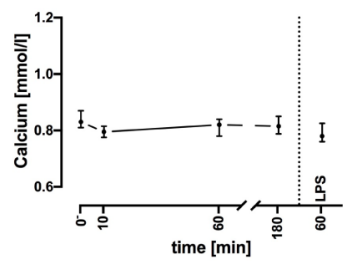

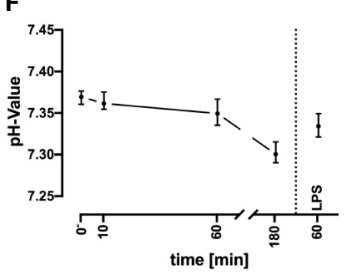

I

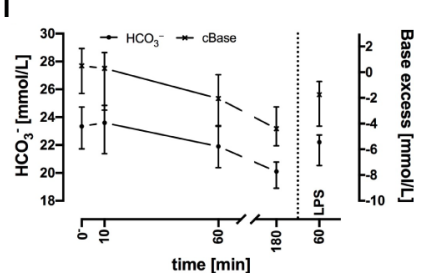

FIGURE 2 | Sodium (A), potassium (B), ionized calcium (C), glucose (D), lactate (E), pH (F), oxygen partial pressure (pO 2 ) (G), carbon dioxide partial pressure $\left(\mathrm{pCO}_{2}\right)(\mathbf{H})$, and acid-base balance (I) before and after exposure of whole blood to the circuit system. Plasma parameters remain widely stable during the first 60 min but show deviations after $3 \mathrm{~h}$. LPS (100 ng/ml) served as PAMP exposure. $\mathrm{n}=3$ for 10 min and 180 min, $n=10$ for all other time points. Results are presented as median with error bars indicating interquartile range.

A

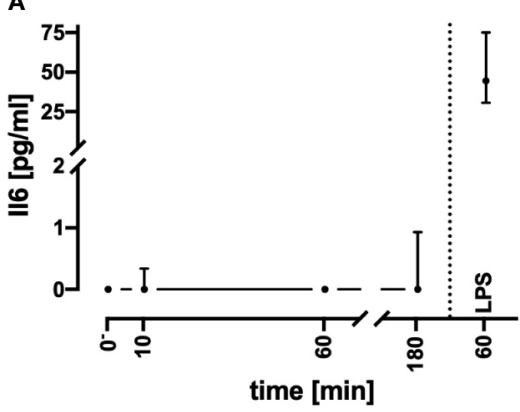

C

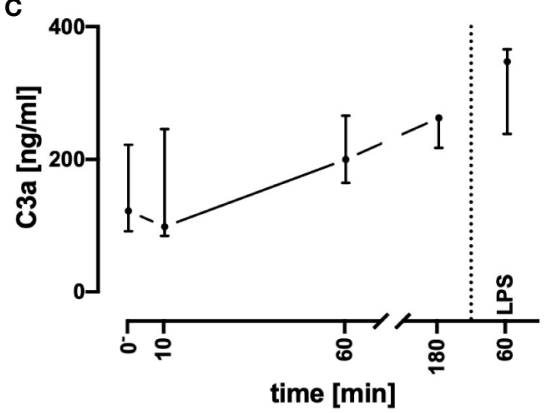

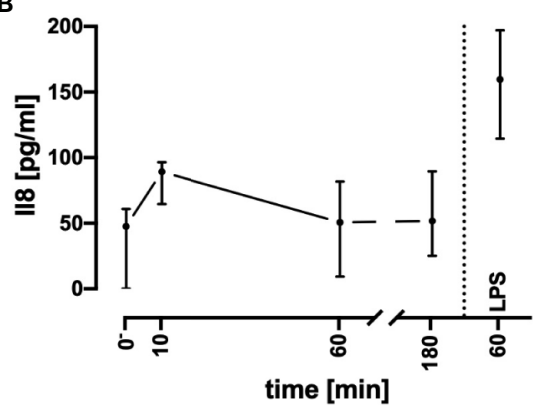

D

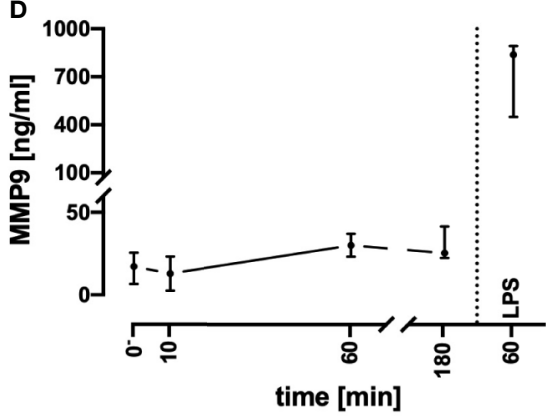

FIGURE 3 | Assessment of humoral inflammatory activity during contact of whole blood with the tubing system by determining the plasma concentrations of II6 (A), II8 (B), C3a (C), and MMP9 (D). Incubation with LPS $100 \mathrm{ng} / \mathrm{ml}$ reflected the presence of PAMPs. $\mathrm{n}=3$ for $10 \mathrm{~min}$ and $180 \mathrm{~min}, \mathrm{n}=10$ for all other time points. Results are presented as median with error bars indicating the interquartile range. 

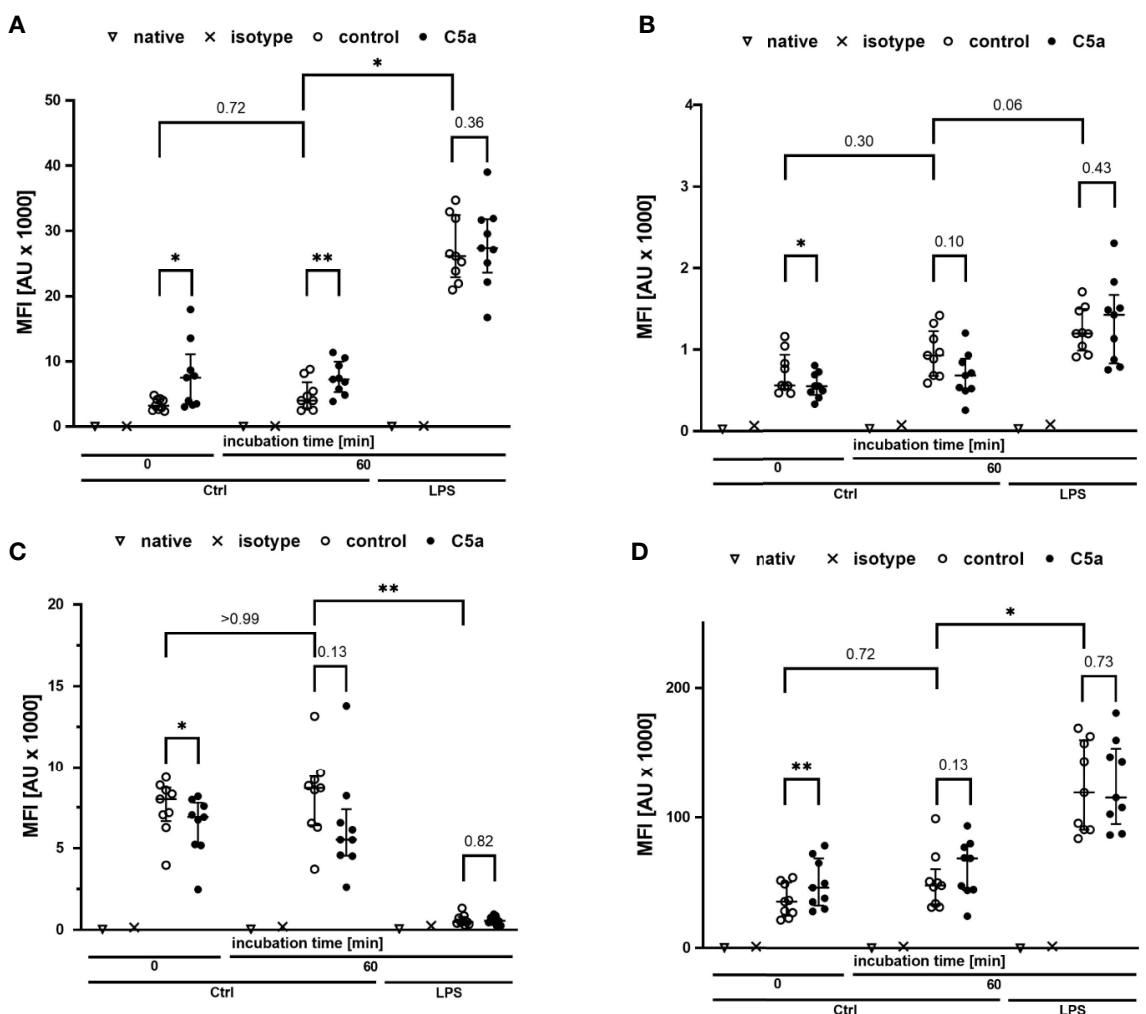

FIGURE 4 | Profiling of activation markers CD11b (A), CD88 (B), CD62 (C), CD14 (D) of monocytes directly after venipuncture (0) and after 60 min of incubation in the whole blood model with or without LPS (100 ng/ml). Following phlebotomy or after the given stimulation period ex vivo, monocytes were stimulated with C5a $(100 \mathrm{ng} / \mathrm{ml})$ in vitro for $15 \mathrm{~min} . \mathrm{n}=9$, results are presented as scatter plot and median with error bars indicating interquartile range. ${ }^{*}=p<0.05,{ }^{\star \star}=p<0.01$.

$16 \%(-12 \% ;-17 \%, \mathrm{n}=10)$, with a glucose consumption rate of $0.80 \mathrm{mmol} / \mathrm{L} / \mathrm{h}(0.60 ; 0.92, \mathrm{n}=10)$. The lactate (Figure $2 \mathrm{E}$ ) concentration almost doubled $(+94 \%, 88 \% ; 126 \%)$ within the first hour, accounting for a generation rate of lactate of 1.4 $\mathrm{mmol} / \mathrm{L} / \mathrm{h}(1.1 ; 1.6, \mathrm{n}=10)$. The $\mathrm{pH}($ Figure $\mathbf{2 F})$ and acid-base balance (Figure 2I) changed accordingly. Partial pressure of oxygen $\left(\mathrm{pO}_{2}\right.$, Figure 2G) increased slightly within the first $10 \mathrm{~min}$, while partial pressure of carbon dioxide $\left(\mathrm{pCO}_{2}\right.$, Figure $\mathbf{2 H}$ ) remained stable. Both parameters indicated that the tubing system was airtight when connected firmly.

\section{Contact With the Tubing System Does Not Trigger an Inflammatory Response}

To assess inflammatory processes induced in the present model system, key inflammatory markers were determined (Figure 3). Of note, the plasma Il8, MMP9, and C3a concentrations remained in a stable range during the monitored incubation period of $3 \mathrm{~h}$. In parallel, Il6 levels were mostly not detectable. Despite the complement system has not been broadly evaluated, it was unlikely that massive $\mathrm{C} 5 \mathrm{a}$ generation occurred. It is established, that neutrophils respond on exposure to C5a with downregulation of CD88 ((31), Figure 5B). However, in the present model system, neutrophil CD88 expression was not significantly altered within the first hour of incubation, indicating excessive complement activity to be unlikely.

\section{Preservation of the Immune and Metabolic Response in the Ex Vivo Situation During the First Hour}

Monitoring of the activation of monocytes and neutrophils was performed by simultaneously analyzing the expression patterns of CD11b, CD88, CD621, and CD14 as well-established markers of the innate immune response after LPS exposure and/or during sepsis. Figures 4, 5 summarize the findings for the incubation period of $1 \mathrm{~h}$, showing no significant alterations after contact within the whole blood model. Additionally, the cellular response pattern of monocytes and neutrophils to in vitro stimulation with C5a either directly after venipuncture or during a defined period in the whole blood model was multi-parametrically evaluated. For all activation markers, there was no relevant change in the cellular response of monocytes or neutrophils before or after incubation in the whole blood model after subsequent in vitro stimulation with $\mathrm{C} 5 \mathrm{a}$.

In addition to the reported extracellular parameters, neutrophils were analyzed regarding the cellular physiology and functionality. As presented in Figures 6A, B, the intracellular $\mathrm{pH}$ and the cellular size of neutrophils were not 
A

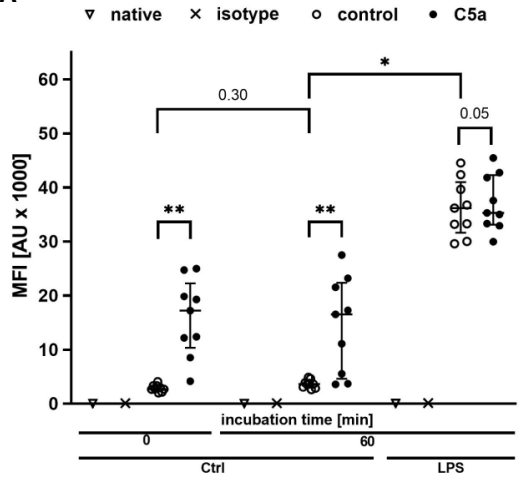

C

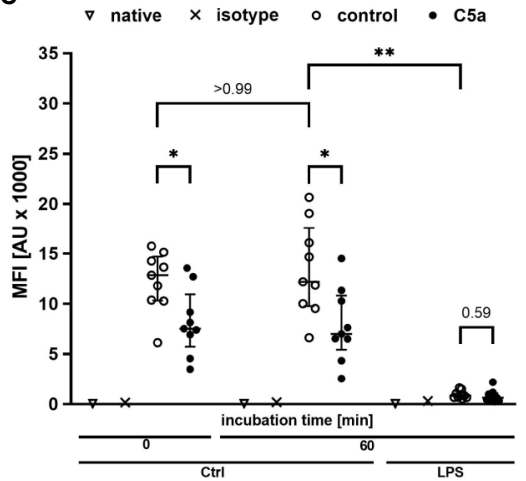

B

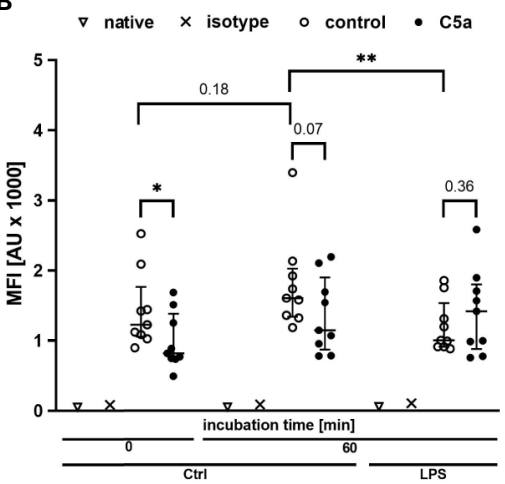

D

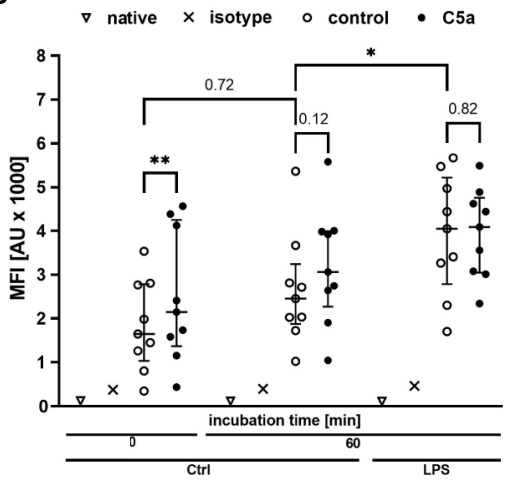

FIGURE 5 | Profiling of the activation markers CD11b (A), CD88 (B), CD621 (C), CD14 (D) of neutrophil granulocytes directly after venipuncture (0) and after 60 min of incubation in the whole blood model with or without LPS $(100 \mathrm{ng} / \mathrm{mll}$ ). Following phlebotomy or after the given stimulation period ex vivo, neutrophils were stimulated with C5a $(100 \mathrm{ng} / \mathrm{ml})$ in vitro for $15 \mathrm{~min} . \mathrm{n}=9$, results are presented as scatter plot and median with error bars indicating the interquartile range. ${ }^{*}=p$ $<0.05,{ }^{\star \star}=p<0.01$.

changed after contact with the whole blood model, apart from a slight intracellular acidification being in accordance with the changes noted in the extracellular $\mathrm{pH}$. Moreover, vital key functions of neutrophil granulocytes remained intact: ROS generation (Figure 6C) and phagocytotic activity (Figure 6D) remained largely stable when comparing neutrophils before and after exposure to the whole blood model, besides a small shift in intracellular $\mathrm{pH}$ and FSC.

\section{Stimulation With LPS Causes Sepsis-Like Changes of the Immunologic Phenotype Ex Vivo}

LPS $(100 \mathrm{ng} / \mathrm{ml})$ was used as a well-described and potent activator of innate immunity, demonstrating that the whole blood model is fully capable of generating a proinflammatory immunologic phenotype. Incubation of the whole blood system with LPS for $1 \mathrm{~h}$ activated the cellular metabolism. Glucose utilization was significantly enhanced by $24 \%$ (15\%; $49 \%$; Ctrl: $-13 \mathrm{mM} / \mathrm{h} ;-16 ;-11$; vs. LPS $-17 \mathrm{mM} / \mathrm{h} ;-20 ;-15 ; p<0.01, \mathrm{n}=$ 10). Similarly, lactate generation was significantly increased by 27\% (22\%; 33\%; Ctrl: $+1.3 \mathrm{mM} / \mathrm{h} ; 1.1 ; 1.6$; vs. LPS: $+1.7 \mathrm{mM} / \mathrm{h}$; $1.5 ; 1.9 ; p<0.01, \mathrm{n}=10)$. Otherwise, global parameters of differential blood count and blood gas analysis remained widely unchanged. As a subsequent step, we compared the C3a, Il6, Il8, and MMP9 levels after stimulation of the whole blood ex vivo with LPS. LPS induced a tremendous increase in Il6 and MMP9, while Il8 and C3a were slightly increased (Figure 3). Interestingly, LPS-induced generation of MMP9 attained a maximum level within the first hour with a concentration that was $10-20$-fold increased after 1 and $3 \mathrm{~h}$ of stimulation in comparison with the control specimens (data not shown). By contrast, LPS-induced generation of Il6 continued to increase beyond $1 \mathrm{~h}$, resulting in a greatly higher level after $3 \mathrm{~h}(1 \mathrm{~h}: 45 \mathrm{pg} /$ $\mathrm{ml} ; 31 ; 75$, vs. 3 h: $2523 \mathrm{pg} / \mathrm{ml} ; 2271 ; 2889$, data not shown, $\mathrm{n}=10$ and $n=3$, respectively).

On the cellular site, monocytes and neutrophils responded to LPS exposure with an increase in the expression rates of CD11 and CD14, while CD62l was markedly decreased. The expression of CD88 was increased in monocytes and decreased in neutrophils after exposure to LPS (Figures 4, 5). Furthermore, the cellular response pattern to additional stimulation in vitro with C5a was significantly impaired. In addition to extracellular activation markers, LPS incubation in the whole blood model altered intracellular parameters of neutrophils, increasing both 

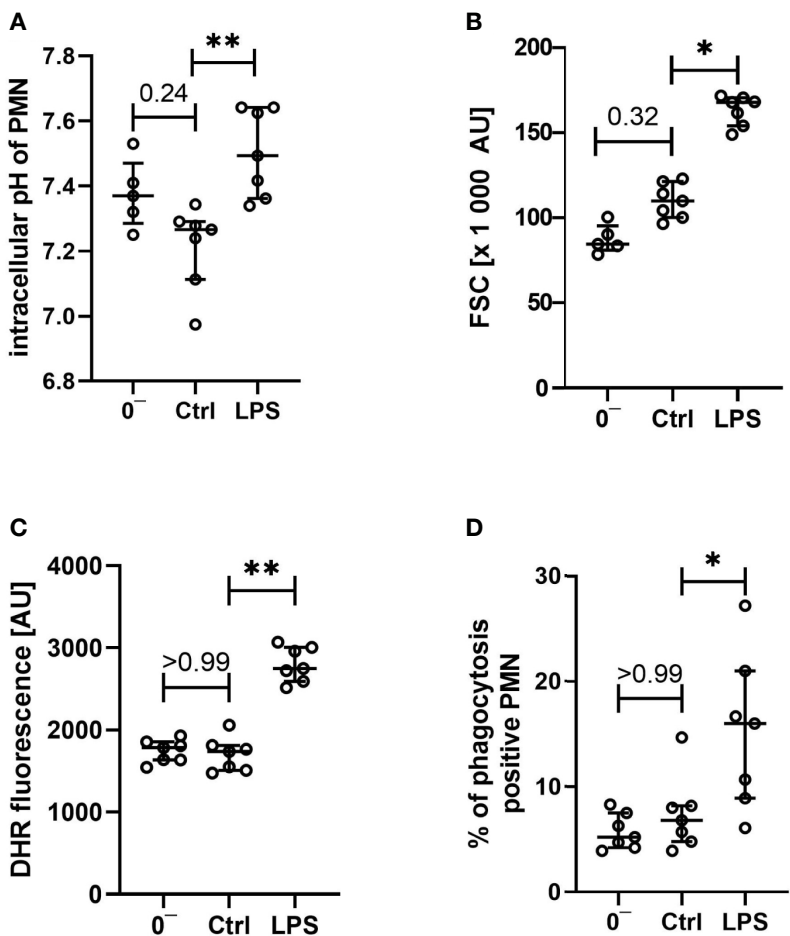

FIGURE 6 | Characterization of intracellular $\mathrm{pH}(\mathbf{A})$, size (B), generation of reactive oxygen species (C), and phagocytotic activity (D) of neutrophils after venipuncture $\left(0^{-}, \mathrm{n}=5-7\right)$ and after incubation in the whole blood model for $1 \mathrm{~h}$ with LPS $100 \mathrm{ng} / \mathrm{ml}$ or without further stimulation (Ctrl, both $\mathrm{n}=7$ ), results are presented as scatter plot and median with error bars indicating the interquartile range. ${ }^{*}=p<0.05,{ }^{\star \star}=p<0.01$

intracellular $\mathrm{pH}$ and size (Figures 6A, B). Moreover, the activity of neutrophils regarding ROS production and phagocytosis was significantly enhanced (Figures 6C, D).

\section{DISCUSSION}

\section{Blood Physiology and Inflammation in Context}

In extracorporeal circulation, the blood system is subjected to various alterations because the interaction with other organ systems is interrupted while in parallel the continuous contact and interaction with the intact endothelium is lost. Furthermore, the ex vivo blood is exposed to artificial surfaces, resulting in activation of thromboinflammation (32). In the current model, the coagulation cascade was inhibited on the material surface and in the blood by a steady level of heparin, however, without administering an extensive amount. Of note, the platelet count and PNC formation remained stable (while still inducible by LPS), indicating no relevant activation of cellular coagulation. Nonetheless, one must consider, that heparins of both high and low molecular weight have been shown to directly interact with immune cells, for example, by increasing myeloperoxidase activity
(25), although, a much lower concentration of heparin $(0.5 \mathrm{IU} / \mathrm{ml})$ was used in the present model. It is also noteworthy, that none of the activation markers assessed in this model were significantly elevated after $1 \mathrm{~h}$ (Figure 7). Regarding metabolic changes of blood physiology, alterations in blood $\mathrm{pH}$, glucose levels, and lactate were noted in the current model. While acidification and lactate levels were tolerable within the first hour, they cannot be considered physiological after $3 \mathrm{~h}$. In a similar manner, blood became hypoglycemic. Cellular innate immunity (particularly neutrophils) mainly relies on anaerobic glycolysis for its metabolism $(33,34)$. Furthermore, cellular effector functions, including phagocytosis, require anaerobic glycolysis (35). Also, hypoglycemia has been shown to aggravate the response to inflammatory stimuli, including LPS (36). In parallel, (lactate) acidosis modulates many activities of the immune system, for example, reducing the phagocytotic capability of neutrophils while increasing their production of MMP9 (37).

Increased Il6 and MMP9 levels are well described in inflammation and during sepsis $(38,39)$. The Il6 levels after $1 \mathrm{~h}$ of stimulation with LPS had almost attained the threshold level of septic patients $(39,40)$. In this regard, it is, however, likely that Il6 during sepsis in realiter can be present longer and attain higher concentrations, because normally more than $1 \mathrm{~h}$ elapses until the patient appears in the clinic with the diagnosis of sepsis. By contrast, the MMP9 levels in the model system were far above the concentrations described in sepsis (38) as early as $1 \mathrm{~h}$ after incubation. It is tempting to speculate that the potent interactions of MMP9 with the glycocalyx of the endothelium, particularly during systemic inflammations that result in degradation of the glycocalyx, which was of course absent in the current ex vivo simulation, may induce higher MMP9 levels ex vivo in comparison with the in vivo situation.

Overall, changes in cellular innate immunity closely resembled the described phenotype alterations in modeled systemic inflammation. For example, in two independent in vivo models of LPS administration in either rats or mice, neutrophil CD62l and CD11b expression appeared similar to the patterns found after LPS exposure in the ex vivo whole blood model $(30,41,42)$. Reduced CD62l expression was also observed in neutrophils from patients with sepsis and endotoxinchallenged human volunteers $(8,43)$. Varying CD11b expression profiles of neutrophils have been reported in clinical sepsis $(43,44)$. These potential differences in CD11b expression (and the differences noted above regarding MMP9 and Il6) may either reflect some limitations of sepsis simulation by isolated LPS stimulation and/or the restricted observation period in the present model. However, the present results regarding the LPS-induced changes of neutrophil expression of CD11b, CD62l, and CD88 are perfectly in line with other whole blood models (45). Additionally, the reduced CD88 expression on leukocytes after simulation of inflammation in the whole blood model is corroborated by data from severely injured, sick, and septic patients (46-49).

In addition, LPS stimulation in the present model resulted in a similar shift of intracellular parameters in comparison with in vivo findings in recent literature. For example, an alkalization of 


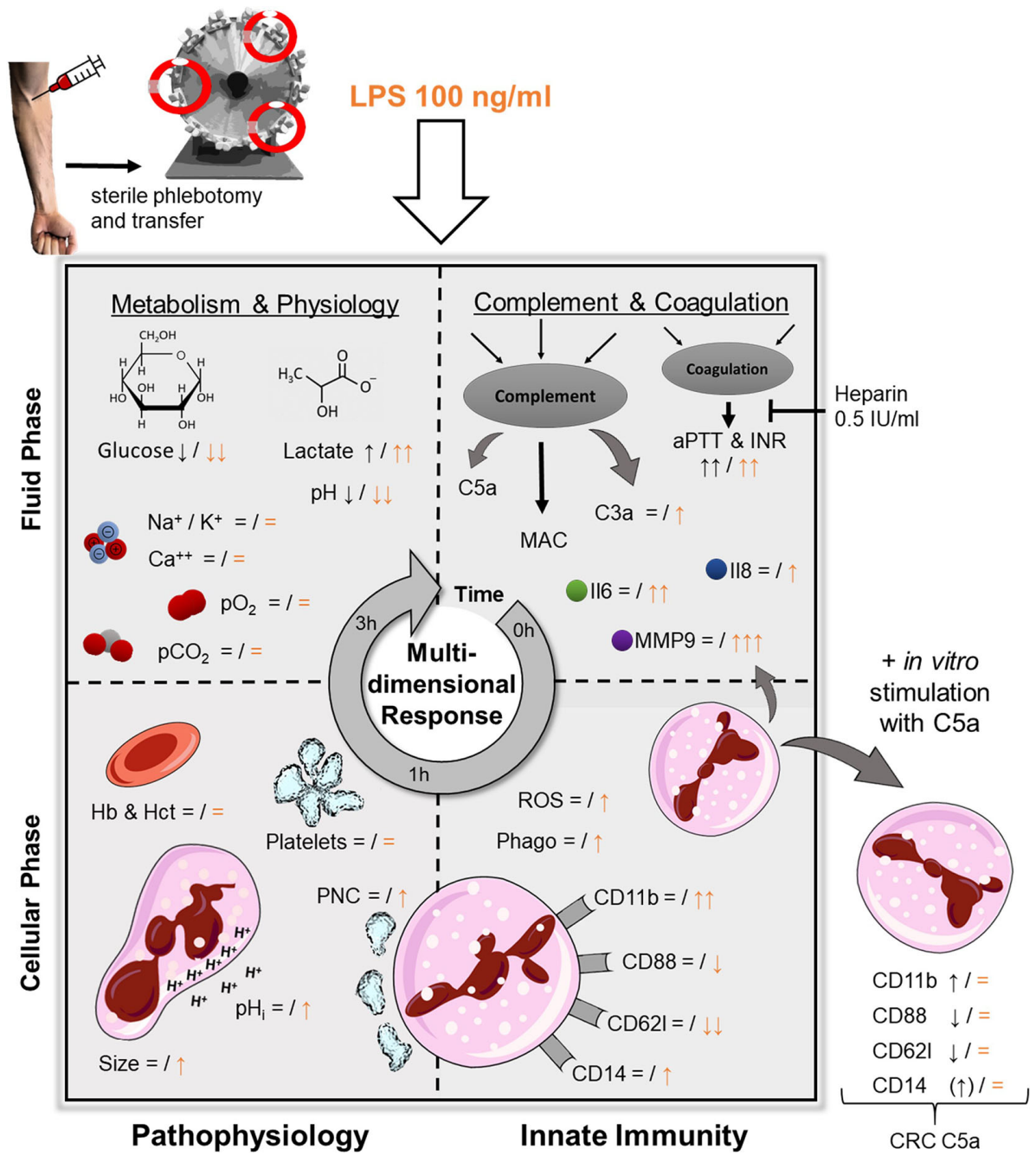

FIGURE 7 | Graphical abstract of the ex vivo whole blood model and the LPS-induced simulation of sepsis. Black symbols represent changes after 1 h of contact with the tubing system, orange indicates a concomitant stimulation with LPS (100 ng/ml). $\mathrm{pO}_{2}$ and $\mathrm{pCO}_{2}$, partial pressure of oxygen and carbon dioxide, respectively; $\mathrm{Hb}$, hemoglobin; Hct, hematocrit; $\mathrm{PNC}$, platelet-neutrophil-complexes; $\mathrm{pH}_{\mathrm{i}}$, intracellular $\mathrm{pH}$; $\mathrm{C} 3 \mathrm{a}$ and $\mathrm{C5a}$, complement factor $3 \mathrm{a}$ and $5 \mathrm{a}$, respectively; MAC, membrane attack complex (sC5b-9); aPTT \& INR, activated partial thromboplastin time and international normalized ratio, respectively; II, Interleukin; MMP9, matrix metallopeptidase 9; ROS, reactive oxygen species; Phago, phagocytosis; CRC, cellular response capacity (to subsequent additional stimulation with C5a.

neutrophils has been reported in septic shock patients (28). Likewise, a rise in size of granulocytes has been described in a murine model of cecum ligation and puncture-induced sepsis (27) and patients after trauma-induced inflammation (50).

\section{Whole Blood Models in Context}

Addressing the complex pathophysiology of systemic inflammation and blood stream infections requires models that can sufficiently portray the various cross-talk mechanisms between the blood cells, the endothelium, the humoral agents of immunity and hemostasis as well as blood physiology in terms of thermoregulation, acid-base-homeostasis, and blood-gas characteristics. Therefore, a plethora of different approaches and protocols were developed to allow the ex vivo investigation of the complex and highly sensitive organ "blood". In this context, the term "whole blood" mostly represents an approach whereby blood, which has been somehow anticoagulated, retains the functionality of most if not, in principle, all its cellular and humoral components. Some protocols apply anticoagulated blood for studies which require an intact immune system, including simulating blood-stream infections (16), analyzing vaccine safety (17), and investigating leukocyte trafficking (51). These models usually add heparin or a hirudin-derived anticoagulant to mainly preserve the complement system as an important calcium-dependent humoral component of innate immunity. By contrast, citrate or Ethylenediaminetetraacetic acid addition interferes with the complement system (32).

Blood models designed to investigate the coagulation cascade, particularly cellular hemostasis, normally use citrated blood including a protocol to resubstitute calcium in a stoichiometric 
manner; however, attempts have been undertaken with hirudinbased models (52). Other groups established a closed in-vivo-like setup by combining defined cell layers or cell culture-based organoids with whole blood $(53,54)$.

\section{Ex Vivo Incubation of Human Whole Blood-Advantages and Limitations}

The presented animal-free ex vivo whole blood model has various advantages: First, it is effortlessly transferable, widely accessible, and convenient to set up, because all the components are commercially available and can be delivered under sterile conditions. Moreover, the materials do not require any special treatment, for example, coating by the user, thus improving standardization and reducing the risk of any contamination. Second, particularly within the first hour, whole blood incubated in the circuit system remains stable regarding both blood physiology and cellular innate immunity. Third, the model allows incubation of several milliliters of blood, enabling the collection of a sufficient amount of specimen for endpoint analysis. The application of an ex vivo stimulation in general allows inclusion of older, frailer individuals, because they are generally underrepresented in inflammatory studies, especially in in vivo LPS challenges (9). Fourth, mimicking the inflammatory stimulus of sepsis with LPS resulted in an immunologic phenotype in accordance with preexisting literature. Last and most importantly, such a reliable model may help support disseminating the 3R-principles of animal research (7) by offering a valid reduction and/or replacement strategy for the investigation of blood-borne and blood-transferred inflammatory and infectious responses.

There are also some limitations of the model. One restriction is the requirement of anticoagulation to reduce the activation of the blood while drawing and transferring it as well as presumably because of the shedding of at least some of the heparin coating into the system. Other whole blood models use different anticoagulants other than heparin, for example, hirudin, which inhibits the coagulation cascade further downstream than heparin. However, because of to the costs of hirudin-based drugs (e.g. lepirudin), and to adhere to the same substance as used for the coating of the tubing, we decided to apply a minimal dose of heparin systemically in the present model. Furthermore, the circulation of the continuously rotating air bubble only partially imitates the circulation forces in the human body without imitating the heterogeneous changes in $\mathrm{pO}_{2}, \mathrm{CO}_{2}$, or flow kinetics in the arterial and venous phases of circulation. Therefore, the changes in $\mathrm{pO}_{2}$ and the stability of $\mathrm{CO}_{2}$ are because of gas exchange with the remaining air bubble in the system, maintaining stable blood gas levels and being comparable to the venous environment. Another limitation is that the simulation of systemic inflammation occurs outside the human body, thereby excluding the blood-organ crosstalk, particularly the interactions with the endothelium, bone marrow, liver, and spleen. However, the current ex vivo setting allows the examination of inflammatory processes uniquely occurring in the blood itself. Finally, the timeframe for analyzing "physiologic" blood ex vivo is limited, because, for example, blood glucose is depleted and metabolic byproducts like lactate are not cleared. However, LPS-induced changes in the phenotype of neutrophils is a rather fast process, that starts within minutes and reached its ceiling as measured by CD62l and CD11b expression on neutrophils within $1 \mathrm{~h}$ (30). Future development might overcome these issues, for example, by supplementing glucose during the experimental course, by connecting the system to a pulsatile pump allowing the circulation of the blood through various organoids such as liver or kidney cells. In addition, although the model in its current setup is easy to perform, a desirable automatization process of the blood handling would be rather complex to achieve.

Overall, based on the data obtained from the synchronous monitoring of a comprehensive arsenal of physiological, metabolic, and immunologic blood parameters in an animalfree environment, we propose an ex vivo whole blood model of sepsis as a valuable addition to in vivo and clinical studies. Further studies need to validate the advantages of this model system in other inflammatory conditions and with blood from patients, for example, to test immunomodulatory treatments in blood from patients with systemic inflammation, cancer, or other diseases without putting patients at risk. Also, this model can be used as a screening tool, which may help to further reduce and/or replace animal experiments.

\section{DATA AVAILABILITY STATEMENT}

The raw data supporting the conclusions of this article will be made available by the authors, without undue reservation.

\section{ETHICS STATEMENT}

The studies involving human participants were reviewed and approved by: Local Independent Ethics Committee of the University of Ulm. The participants provided their written informed consent to participate in this study.

\section{AUTHOR CONTRIBUTIONS}

Conceptualization and supervision: DM and MH-L. Data curation and formal analysis: DM, LV, ME, AS, JB, SH, and MH-L. Methodology: DM, CB, AA, KN, BN, and MH-L. Validation and visualization: DM, LV, and ME. Writingoriginal draft: DM, CB, and MH-L. Writing-reviewing and editing. All authors contributed to the article and approved the submitted version.

\section{FUNDING}

The present work was funded by a research grant ("Forum Gesundheitsstandort") of the Ministry of Science, Research, and Art Baden Wuerttemberg to DM and MH-L, a start-up 
grant and a position as Clinician Scientist to DM by the Collaborative Research Center 1149, project number 251293561, German Research Foundation, as well as grants to KN and BN (grants 2018- 04199, 2016-2075-5.1, and 201604519) provided by the Swedish Research Council. The funders had no role in the design of this study, data collection and interpretation, or decision to submit results.

\section{REFERENCES}

1. Huber-Lang M, Lambris JD, Ward PA. Innate immune responses to trauma. Nat Immunol (2018) 19:327-41. doi: 10.1038/s41590-018-0064-8

2. Singer M, Deutschman CS, Seymour CW, Shankar-Hari M, Annane D, Bauer $\mathrm{M}$, et al. The Third International Consensus Definitions for Sepsis and Septic Shock (Sepsis-3). JAMA (2016) 315:801. doi: 10.1001/jama.2016.0287

3. Rudd KE, Johnson SC, Agesa KM, Shackelford KA, Tsoi D, Kievlan DR, et al. Global, regional, and national sepsis incidence and mortality, 1990-2017: analysis for the Global Burden of Disease Study. Lancet (2020) 395:200-11. doi: 10.1016/S0140-6736(19)32989-7

4. Poli-de-Figueiredo LF, Garrido AG, Nakagawa N, Sannomiya P. EXPERIMENTAL MODELS OF SEPSIS AND THEIR CLINICAL RELEVANCE. Shock (2008) 30:53-9. doi: 10.1097/SHK.0b013e318181a343

5. Fink MP. Animal models of sepsis. Virulence (2014) 5:143-53. doi: 10.4161/ viru. 26083

6. Osuchowski MF, Ayala A, Bahrami S, Bauer M, Boros M, Cavaillon J-M, et al. Minimum Quality Threshold in Pre-Clinical Sepsis Studies (MQTiPSS): An International Expert Consensus Initiative for Improvement of Animal Modeling in Sepsis. SHOCK (2018) 50:377-80. doi: 10.1097/ SHK.0000000000001212

7. Russell WMS, Burch RL. The principles of humane experimental technique. Potters Bar: UFAW (1992). Special ed.

8. Pillay J, Kamp VM, van Hoffen E, Visser T, Tak T, Lammers J-W, et al. A subset of neutrophils in human systemic inflammation inhibits $\mathrm{T}$ cell responses through Mac-1. J Clin Invest (2012) 122:327-36. doi: 10.1172/ JCI57990

9. Fullerton JN, Segre E, De Maeyer RPH, Maini AAN, Gilroy DW. Intravenous Endotoxin Challenge in Healthy Humans: An Experimental Platform to Investigate and Modulate Systemic Inflammation. J Vis Exp (2016). doi: $10.3791 / 53913$

10. Grondman I, Arts RJW, Koch RM, Leijte GP, Gerretsen J, Bruse N, et al. Frontline Science: Endotoxin-induced immunotolerance is associated with loss of monocyte metabolic plasticity and reduction of oxidative burst. J Leukoc Biol (2019) 106:11-25. doi: 10.1002/JLB.5HI0119-018R

11. Hamilos M, Petousis S, Parthenakis F. Interaction between platelets and endothelium: from pathophysiology to new therapeutic options. Cardiovasc Diagn Ther (2018) 8:568-80. doi: 10.21037/cdt.2018.07.01

12. Mollnes TE, Brekke O-L, Fung M, Fure H, Christiansen D, Bergseth G, et al. Essential role of the C5a receptor in E coli-induced oxidative burst and phagocytosis revealed by a novel lepirudin-based human whole blood model of inflammation. Blood (2002) 100:1869-77.

13. Sprong T, Brandtzaeg P, Fung M, Pharo AM, Høiby EA, Michaelsen TE, et al. Inhibition of C5a-induced inflammation with preserved C5b-9-mediated bactericidal activity in a human whole blood model of meningococcal sepsis. Blood (2003) 102:3702-10. doi: 10.1182/blood-2003-03-0703

14. Brekke O-L, Christiansen D, Fure H, Fung M, Mollnes TE. The role of complement C3 opsonization, C5a receptor, and CD14 in E. coli-induced upregulation of granulocyte and monocyte CD11b/CD18 (CR3), phagocytosis, and oxidative burst in human whole blood. J Leukoc Biol (2007) 81:1404-13. doi: $10.1189 / \mathrm{jlb} .0806538$

15. Egge KH, Thorgersen EB, Lindstad JK, Pharo A, Lambris JD, Barratt-Due A, et al. Post challenge inhibition of C3 and CD14 attenuates Escherichia coliinduced inflammation in human whole blood. Innate Immun (2014) 20:6877. doi: $10.1177 / 1753425913482993$

16. Hünniger K, Lehnert T, Bieber K, Martin R, Figge MT, Kurzai O. A Virtual Infection Model Quantifies Innate Effector Mechanisms and Candida albicans

\section{ACKNOWLEDGMENTS}

The authors are indebted to Carina Kleimaier and Anke Schultze for outstanding technical assistance. In addition, the authors are grateful to the Department of Clinical Chemistry, University Hospital of Ulm, Ulm, Germany, for the measurement of differential blood count and coagulation parameters.

Immune Escape in Human Blood. PLoS Comput Biol (2014) 10:e1003479. doi: 10.1371/journal.pcbi.1003479

17. Hakimi J, Aboutorabian S, To F, Ausar SF, Rahman N, Brookes RH. Screening Vaccine Formulations in Fresh Human Whole Blood. Methods Mol Biol (2017) 1494:295-304. doi: 10.1007/978-1-4939-6445-1_22

18. Halbgebauer R, Kellermann S, Schäfer F, Weckbach S, Weiss M, Barth E, et al. Functional immune monitoring in severely injured patients-A pilot study. Scand J Immunol (2020) 91:e12837. doi: 10.1111/sji.12837

19. Wang JE, Solberg R, Okkenhaug C, Jørgensen PF, Krohn CD, Aasen AO. Cytokine modulation in experimental endotoxemia: characterization of an ex vivo whole blood model. Eur Surg Res (2000) 32:65-73. doi: 10.1159/ 000008743

20. Bexborn F, Engberg AE, Sandholm K, Mollnes TE, Hong J, Nilsson Ekdahl K. Hirudin versus heparin for use in whole blood in vitro biocompatibility models. J BioMed Mater Res (2009) 89A:951-9. doi: 10.1002/jbm.a.32034

21. Asif S, Asawa K, Inoue $Y$, Ishihara K, Lindell B, Holmgren R, et al. Validation of an MPC Polymer Coating to Attenuate Surface-Induced Crosstalk between the Complement and Coagulation Systems in Whole Blood in In Vitro and In Vivo Models. Macromol Biosci (2019) 19:1800485. doi: 10.1002/ mabi.201800485

22. World Medical Association Declaration of Helsinki. Ethical Principles for Medical Research Involving Human Subjects. JAMA (2013) 310:2191. doi: 10.1001/jama.2013.281053

23. Dhingra NSafe Injection Global Network and World Health Organization. WHO guidelines on drawing blood: best practices in phlebotomy (2010). Available at: http://www.ncbi.nlm.nih.gov/books/NBK138650/ (Accessed November 10, 2018).

24. Gong J, Larsson R, Ekdahl KN, Mollnes TE, Nilsson U, Nilsson B. Tubing loops as a model for cardiopulmonary bypass circuits: Both the biomaterial and the blood-gas phase interfaces induce complement activation in an in vitro model. J Clin Immunol (1996) 16:222-9. doi: 10.1007/BF01541228

25. Videm V. Heparin in clinical doses "primes" granulocytes to subsequent activation as measured by myeloperoxidase release. Scand J Immunol (1996) 43:385-90. doi: 10.1046/j.1365-3083.1996.d01-57.x

26. Borgdorff P, Tangelder GJ. Pump-induced platelet aggregation with subsequent hypotension: Its mechanism and prevention with clopidogrel. J Thoracic Cardiovasc Surg (2006) 131:813-821.e2. doi: 10.1016/j.jtcvs.2005.10.021

27. Denk S, Taylor RP, Wiegner R, Cook EM, Lindorfer MA, Pfeiffer K, et al. Complement C5a-induced changes in neutrophil morphology during inflammation. Scand J Immunol (2017) 86:143-55. doi: 10.1111/sji.12580

28. Denk S, Neher MD, Messerer DAC, Wiegner R, Nilsson B, Rittirsch D, et al. Complement C5a Functions as a Master Switch for the $\mathrm{pH}$ Balance in Neutrophils Exerting Fundamental Immunometabolic Effects. I Immunol (2017) 198:4846-54. doi: 10.4049/jimmunol.1700393

29. Mauler M, Seyfert J, Haenel D, Seeba H, Guenther J, Stallmann D, et al. Platelet-neutrophil complex formation-a detailed in vitro analysis of murine and human blood samples. J Leukocyte Biol (2016) 99:781-9. doi: 10.1189/ jlb.3TA0315-082R

30. Vlkova M, Chovancova Z, Nechvatalova J, Connelly AN, Davis MD, Slanina P, et al. Neutrophil and Granulocytic Myeloid-Derived Suppressor CellMediated $\mathrm{T}$ Cell Suppression Significantly Contributes to Immune Dysregulation in Common Variable Immunodeficiency Disorders. J Immunol (2019) 202:93-104. doi: 10.4049/jimmunol.1800102

31. van den Berg CW, Tambourgi DV, Clark HW, Hoong SJ, Spiller OB, McGreal EP. Mechanism of Neutrophil Dysfunction: Neutrophil Serine Proteases Cleave and Inactivate the C5a Receptor. JI (2014) 192:1787-95. doi: 10.4049/jimmunol.1301920 
32. Ekdahl KN, Teramura Y, Hamad OA, Asif S, Duehrkop C, Fromell K, et al. Dangerous liaisons: complement, coagulation, and kallikrein/kinin cross-talk act as a linchpin in the events leading to thromboinflammation. Immunol Rev (2016) 274:245-69. doi: 10.1111/imr.12471

33. Kramer PA, Ravi S, Chacko B, Johnson MS, Darley-Usmar VM. A review of the mitochondrial and glycolytic metabolism in human platelets and leukocytes: Implications for their use as bioenergetic biomarkers. Redox Biol (2014) 2:206-10. doi: 10.1016/j.redox.2013.12.026

34. Loftus RM, Finlay DK. Immunometabolism: Cellular Metabolism Turns Immune Regulator. J Biol Chem (2016) 291:1-10. doi: 10.1074/jbc.R115.693903

35. Borregaard N, Herlin T. Energy metabolism of human neutrophils during phagocytosis. J Clin Invest (1982) 70:550-7.

36. Ratter JM, Rooijackers HMM, Tack CJ, Hijmans AGM, Netea MG, de Galan BE, et al. Proinflammatory Effects of Hypoglycemia in Humans With or Without Diabetes. Diabetes (2017) 66:1052-61. doi: 10.2337/db16-1091

37. Erra Díaz F, Dantas E, Geffner J. Unravelling the Interplay between Extracellular Acidosis and Immune Cells. Mediators Inflamm (2018) 2018:1-11. doi: 10.1155/2018/1218297

38. Mühl D, Nagy B, Woth G, Falusi B, Bogár L, Weber G, et al. Dynamic changes of matrix metalloproteinases and their tissue inhibitors in severe sepsis. J Crit Care (2011) 26:550-5. doi: 10.1016/j.jcrc.2011.02.011

39. Song J, Park DW, Moon S, Cho H-J, Park JH, Seok H, et al. Diagnostic and prognostic value of interleukin-6, pentraxin 3, and procalcitonin levels among sepsis and septic shock patients: a prospective controlled study according to the Sepsis-3 definitions. BMC Infect Dis (2019) 19:968. doi: 10.1186/s12879019-4618-7

40. Gentile LF, Cuenca AG, Vanzant EL, Efron PA, McKinley B, Moore F, et al. Is there value in plasma cytokine measurements in patients with severe trauma and sepsis? Methods (2013) 61:3-9. doi: 10.1016/j.ymeth.2013.04.024

41. van Wessem KJP, Heeres M, Leliefeld PHC, Koenderman L, Leenen LPH. Lipopolysaccharide and hemorrhagic shock cause systemic inflammation by different mechanisms. J Trauma Acute Care Surg (2013) 74:37-44. doi: 10.1097/TA.0b013e3182789489

42. Reber LL, Gillis CM, Starkl P, Jönsson F, Sibilano R, Marichal T, et al. Neutrophil myeloperoxidase diminishes the toxic effects and mortality induced by lipopolysaccharide. J Exp Med (2017) 214:1249-58. doi: 10.1084/ jem.20161238

43. Demaret J, Venet F, Friggeri A, Cazalis M-A, Plassais J, Jallades L, et al. Marked alterations of neutrophil functions during sepsis-induced immunosuppression. J Leukocyte Biol (2015) 98:1081-90. doi: 10.1189/jlb.4A0415-168RR

44. Groeneveld KM, Koenderman L, Warren BL, Jol S, Leenen LPH, Hietbrink F. Early decreased neutrophil responsiveness is related to late onset sepsis in multitrauma patients: An international cohort study. PLoS One (2017) 12: e0180145. doi: 10.1371/journal.pone.0180145

45. Maini AA, George MJ, Motwani MP, Day RM, Gilroy DW, O’Brien AJ. A Comparison of Human Neutrophils Acquired from Four Experimental
Models of Inflammation. PLoS One (2016) 11:e0165502 doi: 10.1371/ journal.pone.0165502

46. Furebring M, Håkansson LD, Venge P, Nilsson B, Sjölin J. Expression of the C5a receptor (CD88) on granulocytes and monocytes in patients with severe sepsis. Crit Care (2002) 6:363-70. doi: 10.1186/cc1524

47. Amara U, Kalbitz M, Perl M, Flierl MA, Rittirsch D, Weiss M, et al. Early Expression Changes of Complement Regulatory Proteins and C5a Receptor (Cd88) On Leukocytes After Multiple Injury In Humans. Shock (2010) 33:568-75. doi: 10.1097/SHK.0b013e3181c799d4

48. Morris AC, Brittan M, Wilkinson TS, McAuley DF, Antonelli J, McCulloch C, et al. C5a-mediated neutrophil dysfunction is RhoA-dependent and predicts infection in critically ill patients. Blood (2011) 117:5178-88. doi: 10.1182/ blood-2010-08-304667

49. Unnewehr H, Rittirsch D, Sarma JV, Zetoune F, Flierl MA, Perl M, et al. Changes and Regulation of the C5a Receptor on Neutrophils during Septic Shock in Humans. J Immunol (2013) 190:4215-25. doi: 10.4049/ jimmunol.1200534

50. Hesselink L, Heeres M, Paraschiakos F, ten Berg M, Huisman A, Hoefer IE, et al. A Rise in Neutrophil Cell Size Precedes Organ Dysfunction After Trauma. SHOCK (2019) 51:439-46. doi: 10.1097/SHK.0000000000001200

51. Hamza B, Irimia D. Whole blood human neutrophil trafficking in a microfluidic model of infection and inflammation. Lab Chip (2015) 15:2625-33. doi: 10.1039/c5lc00245a

52. Tiedemann Skipper M, Rubak P, Halfdan Larsen O, Hvas A-M. Thrombocytopenia model with minimal manipulation of blood cells allowing whole blood assessment of platelet function. Platelets (2016) 27:295-300. doi: 10.3109/09537104.2015.1095873

53. Schmohl M, Schneiderhan-Marra N, Baur N, Hefner K, Blum M, Stein GM, et al. Characterization of immunologically active drugs in a novel organotypic co-culture model of the human gut and whole blood. Int Immunopharmacol (2012) 14:722-8. doi: 10.1016/j.intimp.2012.10.010

54. Nordling S, Nilsson B, Magnusson PU. A novel in vitro model for studying the interactions between human whole blood and endothelium. J Vis Exp (2014), e52112. doi: 10.3791/52112

Conflict of Interest: The authors declare that the research was conducted in the absence of any commercial or financial relationships that could be construed as a potential conflict of interest.

Copyright (c) 2020 Messerer, Vidoni, Erber, Stratmann, Bauer, Braun, Hug, Adler, Nilsson Ekdahl, Nilsson, Barth, Radermacher and Huber-Lang. This is an open-access article distributed under the terms of the Creative Commons Attribution License (CC BY). The use, distribution or reproduction in other forums is permitted, provided the original author(s) and the copyright owner(s) are credited and that the original publication in this journal is cited, in accordance with accepted academic practice. No use, distribution or reproduction is permitted which does not comply with these terms. 